\title{
Contribution
}

\section{à la surveillance du personnel exposé à des composés d'uranium non transférables $\left(^{*}\right)$}

\author{
J. CAMARASA $(* *)$, J. CHALABREYSSE $(* * *)$ \\ (Reçu le 16 novembre 1979)
}

\begin{abstract}
RÉSUMÉ
Après avoir rappelé brièvement les connaissances actuelles concernant l'uranium (métabolismes, toxicité, principes de surveillance radiotoxicologique), les auteurs décrivent l'expérience acquise dans la surveillance de sujets exposés à des composés d'uranium naturel non transférables (oxydes, tétrafluorure). Ils présentent les difficultés qu'ils ont rencontrées pour la mise en place des contrôles urinaires parmi une population de travailleurs, dans la façon de prélever les urines, dans l'obtention de la pureté de l'échantillon, dans l'interprétation des résultats.

Parallèlement aux contrôles urinaires, des études d'ambiance de travail sont effectuées; les mesures de la pollution sont de trois types (ambiance générale à poste fixe avec mesure de la radioactivité du prélèvement, poste de travail et travailleur lui-même, par mesure chimique et par comptage de l'uranium présent). Des courbes originales de surveillance des postes de travail sont régulièrement tenues à jour. A l'heure actuelle, la surveillance des travailleurs par les contrôles urinaires et celle des conditions de travail sont bien codifiées. Néanmoins, d'autres études sont prévues sur l'homme, sur les ambiances de travail, sur les produits manipulés. Cette surveillance couvrira ainsi tous les aspects des conditions de travail.
\end{abstract}

\begin{abstract}
After a short review of the present knowledge on uranium (metabolism, toxicity, principles of radiotoxicological monitoring), the authors' experience in the surveillance of workers exposed to natural non-transferable uranium
\end{abstract}

(*) De cette étude a été tirée une communication présentée aux $15^{\text {e }}$ Journées nationales de Médecine du Travail de Strasbourg, 10 au 13 mai 1978.

(**) Médecine du Travail, Société Comurhex, B. P. n 70, 11101 Narbonne.

$\left({ }^{* * *}\right)$ Commissariat à l'Énergie atomique, I.P.S.N., Département de Protection, Service d'hygiène industrielle, B. P. $n^{\circ} 38,26700$ Pierrelatte. 
compounds (oxides, tetrafluorides) is presented. When setting up urinary controls in a workers' population, a number of difficulties were met in the way of collecting urine samples, obtaining samples free of exogen contribution, interpreting results.

The working environment was also studied: three types of pollution measurements were carried out: on the atmosphere at fixed places by measuring the radioactivity of air sample, on work-places and workers by chemical analysis and counting of uranium. Original graphs on work-place monitoring are up-dated regularly. Workers' surveillance by urinary and working condition controls are now well codified. However, further studies will be carried out on man, on working atmospheres, and on the substances handled. The surveillance will then cover working conditions from all points of view.

\section{PRÉAMBULE}

Si la surveillance de l'irradiation externe est relativement aisée et bien codifiée, il n'en est pas de même de celle de la contamination interne toujours difficile à déterminer, en particulier dans le cas des poussières uranifères. Cette surveillance ne peut se faire bien souvent que d'une manière indirecte, par la mesure de l'excrétion urinaire ou les dosages dans les fèces, l'anthropogammamétrie, par la mesure directe du rayonnement gamma, nécessitant des installations complexes.

En usine, dans le cas qui nous intéresse plus particulièrement, nous avons été amenés, dès 1959, à surveiller l'excrétion urinaire de l'uranium chez les travailleurs exposés aux composés dits insolubles $\left({ }^{1}\right)$. Notre surveillance était basée sur deux notions fournies par la littérature scientifique :

- une première notion de " niveau d'imprégnation " qu'il convenait de ne pas dépasser et qui paraissait correspondre à des doses non préjudiciables à l'organisme;

- une deuxième notion de période biologique.

La première notion a été confirmée rapidement : les résultats enregistrés ne paraissaient pas préjudiciables à la santé des travailleurs (pas d'atteinte de l'hémogramme, de la fonction rénale et du parenchyme pulmonaire). Par contre, la deuxième notion ne parut pas correspondre à la réalité et nous eûmes rapidement l'impression que les composés " insolubles " auxquels nous avions affaire s'éliminaient suivant des périodes biologiques beaucoup plus courtes que ne le laissait prévoir la littérature. Ceci demandait à être vérifié. Par ailleurs, il apparût nécessaire de codifier de façon plus rigoureuse la surveillance systématique à mesure qu'augmentaient la production et le nombre de travailleurs à surveiller. Ces deux raisons nous ont amenés à critiquer systématiquement nos différentes techniques et à essayer de mieux codifier la surveillance du personnel. Nous nous sommes ainsi aperçus que de multiples paramètres pouvaient intervenir tant dans les prélèvements urinaires que dans

( $\left.{ }^{1}\right)$ On différenciait alors les composés "solubles » qui s'éliminaient en quelques jours et les composés " insolubles " dont la période d'élimination variait, suivant les auteurs, entre 200 et 500 jours. 
les techniques de laboratoire et pouvaient ainsi fausser les résultats; nous les avons corrigés au fur et à mesure de leur mise en évidence. Nous avons ainsi été amené à reconsidérer les résultats qui nous étaient fournis. Nous somme alors arrivés à la notion qu'un seul chiffre avait peu de signification et qu'il devait être intégré dans un ensemble, étudié sur graphiques, cet ensemble englobant le plus grand nombre possible de données, se rapprochant ellesmêmes, le plus possible, d'une réalité bien difficile à déterminer.

\section{INTRODUCTION}

Cette communication expose les diverses étapes qui ont conduit progressivement à la mise au point d'une méthode de surveillance du personnel dans une usine d'uranium (Comurhex-Malvésy, Narbonne). Depuis sa mise en service, l'usine produit du tétrafluorure d'uranium à partir des différents composés uranifères qui lui sont livrés. Une partie du tétrafluorure est ensuite transformée en uranium métal, par magnésiothermie, l'autre est expédiée dans une autre usine pour fabrication de l'hexafluorure.

L'exposition des travailleurs aux poussières uranifères est le risque majeur. Elle rend nécessaire une surveillance permanente et systématique des conditions de travail et des travailleurs eux-mêmes; elle s'exerce, en moyenne, sur 200 salariés par an, depuis 20 ans.

\section{BIOTOXICOLOGIE DE L'URANIUM}

Sans entrer dans des considérations déjà bien connues, nous rappellerons néanmoins quelques notions essentielles sur l'uranium et sa biotoxicologie.

2.1. L'URANIUM, ÉLÉMENT DE NUMÉRo ATOMIQUE 92 existe dans la nature sous forme d'un mélange de trois isotopes $(238,235,234)$, dans les proportions respectives en masse sont de $99,28,0,72$ et 0,0056 p. cent. Les périodes radioactives de chacun de ces isotopes sont très longues, de l'ordre de plusieurs millions d'années. Ils sont tous trois émetteurs de particules alpha et de rayonnement gamma. A l'état naturel, l'uranium est très faiblement radioactif (1 $\mu \mathrm{g}$ d'uranium correspond à $1,5 \mathrm{dpm}$ ).

Selon la Commission Internationale de Protection Radiologique (C.I.P.R.) on peut classer les composés d'uranium en trois catégories selon leur comportement métabolique :

Composés transférables (classe D) dont la période biologique d'épuration au niveau du poumon est de l'ordre de la journée $(0,5 \mathrm{j})$. Ce sont l'hexafluorure $\mathrm{UF}_{6}$; l'oxyfluorure $\mathrm{UO}_{2} \mathrm{~F}_{2}$; le nitrate d'uranyle $\mathrm{UO}_{2}\left(\mathrm{NO}_{3}\right), 6 \mathrm{H}_{2} \mathrm{O}$.

Composés moyennement transférables (classe $\mathrm{W}$ ) dont la période biologique d'épuration s'étend sur des semaines $(50 \mathrm{j})$. Ce sont le tétrafluorure $\mathrm{UF}_{4}$; le trioxyde $\mathrm{UO}_{3}$; le diuranate d'ammonium $\left(\mathrm{NH}_{4}\right)_{2} \mathrm{U}_{2} \mathrm{O}_{7}$; le péruranate $\mathrm{Na}_{2} \mathrm{U}_{2} \mathrm{O}_{7}$; le carbonate d'uranyle $\mathrm{UO}_{2} \mathrm{CO}_{3} \mathrm{H}_{2} \mathrm{O}$.

VOL. $15-\mathrm{N}^{\circ} 1$ 
Composés peu transférables (classe $\mathrm{Y}$ ) dont la période biologique d'épuration dépasse l'année $(500 \mathrm{j})$. Ce sont : le dioxyde $\mathrm{UO}_{2} ;$ l'oxyde $\mathrm{U}_{3} \mathrm{O}_{8}$; les carbures $\mathrm{UC}_{1}, \mathrm{UC}_{2}$, $\mathrm{U}_{2} \mathrm{C}_{3}$.

\subsection{Métabolisme de L'URANiUm}

Le métabolisme peut être décomposé schématiquement en trois phases successives : une première qui va de l'absorption du composé jusqu'au passage de l'uranium dans le courant sanguin; ensuite la répartition de l'uranium dans l'organisme, à partir du sang; enfin, son élimination.

\section{Absorption}

Au premier stade du schéma, le caractère de solubilité dans l'eau du composé intervient; par extension, on suppose qu'il en est de même dans les liquides de l'organisme. Dans le cas d'un composé d'uranium transférable (classe D), le passage dans le sang est très rapide. S'il s'agit, par contre, d'un composé d'uranium peu transférable, il faut tenir compte du temps nécessaire à la solubilisation progressive au niveau de la porte d'entrée.

\section{Diffusion dans l'organisme}

Après avoir pénétré dans l'organisme par quelque voie que ce soit, et après un temps de latence au niveau de la porte d'entrée, variable suivant le degré de solubilité du composé, l'uranium diffuse dans le sang, puis il se disperse dans le milieu extra-cellulaire et se concentre dans les organes de stockage qui sont pour les composés à la valence $6:$ le squelette et le rein; pour les composés à la valence 4 : le système réticuloendothélial du foie et de la rate.

\section{Élimination}

Mais il n'y a pas fixation définitive et il existe toujours un " relargage » de l'uranium des organes de stockage vers le sang. L'uranium sanguin est très rapidement épuré : $80 \mathrm{p}$. cent de l'uranium parvenu au sang s'éliminent dans les urines des premières 24 heures. Les urines sont donc la voie principale d'élimination de l'uranium. Il existe une autre voie, les selles, où se retrouvent les particules d'uranium inhalées qui n'ont pas atteint l'alvéole pulmonaire et qui, déposées dans les voies respiratoires supérieures, sont évacuées par des processus mécaniques via le tube digestif.

Tardivement, on retrouve aussi dans les selles l'uranium peu transférable qui a quitté le compartiment pulmonaire, en partie par le sang, en partie par les ganglions trachéobronchiques, et qui, après avoir chargé le squelette et les reins, s'élimine par les sécrétions digestives.

\subsection{TOXICITÉ DE L'URANIUM}

Elle revêt plusieurs aspects, selon qu'il se trouve à l'état naturel ou enrichi $\left({ }^{235} \mathrm{U}\right)$, selon que le composé est de type transférable ou peu transférable ou selon que la contamination est unique ou chronique.

Dans le cas qui nous préoccupe, nous avons affaire à des composés d'uranium naturel peu transférables $\left(\mathrm{U}_{3} \mathrm{O}_{8}\right)$ ou moyennement transférables (uranates, $\mathrm{UF}_{4}, \mathrm{UO}_{3}$ ), sous forme de poussières susceptibles d'être inhalées de façon chronique; c'est donc un risque de contamination interne. La toxicité est de nature radiologique, avec pour organe critique le poumon, en raison de la longue durée de rétention des particules au niveau de cet organe. Le rayonnement alpha, émis par l'uranium localisé dans le tissu pulmonaire, entraîne au niveau de la cellule, le désordre habituel des radiations 
ionisantes. Pour ce type de composés, le risque rénal n'existe pratiquement pas, à la différence de ce que l'on peut observer avec les composés d'uranium transférables.

\subsection{Principe de la surveillance}

La surveillance du personnel exposé à des composés d'uranium consiste à s'assurer que sont bien respectées les normes professionnelles d'exposition à ces composés : il s'agit donc d'évaluer les quantités ou activités effectivement absorbées par le travailleur. Pour cela, on peut faire appel à deux procédés :

(a) Surveillance des ambiances de travail. On peut pratiquer trois types de contrôle :

- contrôle d'ambiance générale;

- contrôle de postes de travail;

- contrôle individuel.

(b) Surveillance radiotoxicologique des travailleurs selon deux types de mesures :

- mesure directe de la radioactivité localisée au niveau du poumon de l'individu (anthropogammamétrie);

- mesure indirecte par examens sur des prélèvements biologiques (urines, selles).

\subsection{Méthodes de SURVEILlanCE UTILISÉES A L'USINE}

A l'heure actuelle, en surveillance systématique, le dosage de l'uranium urinaire est la méthode la plus rapide et la plus facile à réaliser. Nous la pratiquons régulièrement, associée aux mesures d'activité des ambiances des ateliers qui sont fournies quotidiennement par le Service de Protection contre les Radiations (SPR) de l'usine. Nous la complétons, pour des études plus approfondies, par les dosages de l'uranium dans les fèces, plus difficiles à obtenir, et par l'anthropogammamétrie qui demande un appareillage important que nous n'avons pas sur place. (Ces différents examens et analyses sont effectués par le Service d'hygiène industrielle (SHI), à Pierrelatte.)

Les résultats des analyses d'excrétion urinaire, sont exprimés en microgrammes par litre d'urine. En 1959, le seuil à ne pas dépasser, en principe, avait été fixé à $80 \mu \mathrm{g}$ par litre d'urine; $50 \mu \mathrm{g}$ étant le niveau moyen au-dessus duquel on s'efforcerait de rester. Nous avons admis que plusieurs dépassements de la limite supérieure ne seraient pas forcément dangereux s'ils étaient espacés dans le temps, mais devraient entraîner une surveillance particulière et éventuellement un changement de poste ou une " mise au vert » du travailleur, si ces dépassements étaient répétés dans un court intervalle de temps, 3 mois environ. Ces valeurs et leur mode d'interprétation demanderaient certainement à être approfondis : elles paraissaient néanmoins correspondre, d'après la littérature scientifique, à des doses non préjudiciables à l'organisme.

VOL. $15-\mathrm{N}^{\circ} 1$ 


\section{PREMIÈRES OBSERVATIONS : COURBES D'EXCRÉTION INDIVIDUELLE ET COURBES DE SUIVI DE POSTE}

De 1960 à 1972, les taux d'excrétion urinaire ont oscillé entre 10 et $40 \mu \mathrm{g}$ par litre, avec parfois des pics isolés à 100 et même $200 \mu \mathrm{g}$ qui revenaient en quelques jours aux valeurs habituellement rencontrées. Le retour à ces valeurs était beaucoup plus rapide que celui auquel on aurait pu s'attendre (jusqu'à 500 j, d'après la C.I.P.R.). Nous avons donc décidé, à partir de 1979, d'étudier, d'une façon beaucoup plus rigoureuse, l'excrétion de l'uranium urinaire; ceci se justifiait d'autant plus que l'augmentation du rythme de production nous imposait une méthode de surveillance plus stricte et mieux codifiée. Mais, pour en arriver là, nous avons rencontré de nombreuses difficultés qu'il nous paraît intéressant d'exposer.

\subsection{Difficultés ReNCONTRÉEs DANS LA DÉTERMination DE L'ORIGINE DE L'URANIUM}

Et tout d'abord quelles sont les origines de l'uranium retrouvé dans les prélèvements d'urine ? Il provient du sang épuré par le rein. Il a donc la même diversité d'origine que l'uranium sanguin, puisque le sang véhicule l'uranium des portes d'entrée vers les organes de stockage ou de ceux-ci vers le rein. Quatre origines sont théoriquement possibles :

(a) L'uranium peut provenir d'une absorption récente de composés inhalés juste avant la prise d'échantillon et pour lesquels le passage à travers la barrière pulmonaire est rapide (composés transférables). Dans ce cas, son déplacement est rapide, il nécessite moins d'une journée. On peut donc s'attendre à ce que le taux d'excrétion urinaire soit maximal au cours des 24 heures suivant l'absorption ( $80 \mathrm{p}$. cent de la quantité ayant atteint les alvéoles pulmonaires sont excrétés dans les urines) et qu'il diminue ensuite rapidement. Ainsi, pour estimer une exposition récente à de tels composés, le meilleur moment pour collecter les urines est la fin de la journée de travail, ou mieux, la fin d'une semaine de travail, le vendredi soir.

(b) L'uranium accumulé dans les poumons, à la suite d'exposition à des composés peu transférables, au cours des mois précédant l'examen, est libéré lentement suivant une période biologique théorique de $500 \mathrm{j}$. L'excrétion se fait à des niveaux sensiblement constants.

(c) Il peut provenir également des dépôts qui se sont effectués dans le rein au cours des semaines précédentes; mais la période effective au niveau de cet organe est faible $(15 \mathrm{j})$ et il ne se fixe que $2,8 \mathrm{p}$. cent de la quantité inhalée. En conséquence, les quantités éliminées à partir de cet organe seront faibles et rapidement décroissantes. 
(d) L'uranium se répartit également dans le squelette; $85 \mathrm{p}$. cent de la quantité totale dans l'organisme sont retenus dans le squelette. Il s'en élimine lentement suivant une période biologique ou effective de $300 \mathrm{j}$.

On conçoit donc la difficulté d'interprétation des examens urinaires. Pour distinguer entre toutes ces origines, nous procédons de la façon suivante :

- un premier prélèvement d'urine est pratiqué en fin d'une semaine de travail $\left({ }^{1}\right)$ : dans cet échantillon, on retrouve à la fois l'uranium récemment absorbé sous forme de composés transférables et l'uranium provenant des organes de stockage (squelette, rein) ou libéré lentement à partir du poumon pour les composés peu transférables et correspondant à des contaminations anciennes;

- un deuxième prélèvement, après un week-end de repos, avant la reprise de travail $\left({ }^{1}\right)$. En principe, il ne contient pas d'uranium transférable, déjà éliminé pendant les 2 ou 3 jours du week-end. Il ne renferme, en principe, que l'uranium provenant des organes de stockage (os, rein), ou provenant du poumon; c'est lui qui correspond à ce que nous appelons " le bruit de fond » et, en réalité, c'est celui qui nous intéresse le plus. Mais pour en arriver là, nous nous sommes heurtés à de nombreuses diffcultés.

\subsection{DifFICULTÉS RENCONTRÉES DANS LES PRÉLÈVEMENTS URINAIRES}

Nous nous sommes demandés si les échantillons d'urine reflétaient bien la réalité de l'imprégnation, car les résultats s'exprimant en microgrammes, le moindre apport exogène fausse le résultat. Ainsi, nous nous sommes aperçus que le fait d'uriner après la douche dans les vestiaires ne donnait pas entière satisfaction, les travailleurs n'étant pas suffisamment décontaminés, d'où risque de souillure de l'échantillon d'urine. De même, une douche prise trop rapidement n'était pas assez abondante et le sujet insuffisamment rincé. C'est ainsi que les prélèvements faits à l'infirmerie ont dû être abandonnés afin d'être absolument certain que tout apport exogène était impossible. Nous avons donc été amenés à demander au personnel d'uriner à domicile; ce fut difficile à réaliser en raison d'oublis fréquents, et nécessita un effort d'information, de compréhension et de patience.

Actuellement, en fin de poste, le travailleur reçoit deux flacons portant les dates auxquelles il doit uriner : le premier, au saut du lit le lendemain de la fin de la période de poste, et le deuxième, au saut du lit avant la reprise, c'est-à-dire 2 à 3 jours après. Avant de reprendre son travail, en début de poste, il dépose à l'infirmerie les deux flacons pleins $(220 \mathrm{ml}$ environ pour chacun) qu'on lui a confiés vides, 2 à 3 jours auparavant. Nous pouvons donc penser que tout apport exogène a été supprimé. Dans le même esprit, nous avons décidé que la préparation des flacons serait faite en dehors de l'usine par le S.H.I., chargé des analyses et théoriquement indemne de toute contamination.

( $\left.{ }^{1}\right)$ Dans chacun des cas, il ne s'agit pas de la totalité des urines de 24 heures, mais nous faisons recueillir, au domicile de chaque travailleur, 150 à $200 \mathrm{ml}$ d'urine.

vol. $15-\mathrm{N}^{\circ} 1$ 


\subsection{Difficultés au niveau du dosage}

Après avoir résolu le problème de l'apport exogène et de la contamination externe du prélèvement, nous nous sommes aperçus qu'il pouvait exister des possibilités d'erreurs dans le dosage même de l'uranium. C'est ainsi que pour un même échantillon que nous avions séparé en deux aliquotes à l'infirmerie, adressées à deux laboratoires différents (Marcoule et Pierrelatte), nous avons reçu des réponses sensiblement différentes et ce, à plusieurs reprises. Par exemple, une aliquote donnait $94 \mu \mathrm{g} / \mathrm{l}$ et l'autre $10 \mu \mathrm{g} / \mathrm{l}$. On comprend donc notre perplexité à la lecture de tels résultats sachant que $50 \mathrm{mg}$ représentent une excrétion urinaire excessive et $10 \mathrm{mg}$ une excrétion dite acceptable. Les laboratoires, après une étude approfondie, ont démontré que leur technique n'était pas en cause. En fait, il se produisait, à l'infirmerie, avant fractionnement de l'échantillon en deux aliquotes, une précipitation des phosphates dans les urines; cette précipitation provoquait une coprécipitation partielle de l'uranium qui était ainsi en concentration plus importante dans le précipité que dans le surnageant limpide. Au moment de la séparation de l'échantillon, l'infirmerie répartissait dans deux flacons différents des liqueurs dont la concentration en uranium était différente; il n'était donc pas étonnant que chaque laboratoire trouve des résultats différents. Ce point particulier, pour mineur qu'il paraisse, mérite d'être signalé, car il est riche d'enseignements :

- les dosages toxicologiques, particulièrement délicats par définition, doivent être confiés à des laboratoires spécialisés;

- tout dosage doit être pratiqué sur une aliquote représentative de l'excrétion, ce qui suppose une bonne homogénéisation avant le fractionnement des urines (dans le cas d'une expédition, par exemple).

\subsection{DifFICULTÉS POUR AVOIR UN PRÉLÈVEMENT REPRÉSENTATIF DE L'EXCRÉTION URINAIRE}

L'interprétation du résultat en fonction de l'élimination individuelle était essentielle, une aide importante nous a été fournie par le dosage de la créatinine urinaire. On sait, en effet, que la quantité de créatinine urinaire est relativement constante pour un individu donné : homme : $2 \pm 0,5 \mathrm{~g} / 24 \mathrm{~h}$; femme : $1,5 \pm 0,5 \mathrm{~g} / 24 \mathrm{~h}$. Par ailleurs, les concentrations urinaires d'un constituant varient au cours de la journée en fonction du volume des liquides ingérés. Mais, étant donné que la quantité de créatinine excrétée dans les 24 heures est à peu près constante, la concentration urinaire de la créatinine reflète bien le taux de concentration ou de dilution de l'urine. En rapprochant le taux de concentration de l'uranium au taux de créatinine urinaire pour le même échantillon, on peut apprécier, avec une plus grande précision, la quantité réelle d'uranium excrétée. Cette façon de procéder nous a permis de mieux comprendre des résultats qui nous paraissaient aberrants à première vue (tableau I). 
Ainsi, chez les sujets AY et RA : taux de créatinine bas le premier jour, excrétion $U_{1}$ et $U_{2}$ paraissant identiques à chaque fois (8-10 et 25-27), en fait, les excrétions rapportées au taux de créatinine sont nettement différentes (19,5 et 4 pour AY et 43 et 11 pour RA).

\section{TABLEAU I}

EXCRÉTION DE L'URANIUM URINAIRE.

COMPARAISON RAPPORTÉE AU VOLUME D'URINE ET A LA QUANTITÉ DE CRÉATININE

\begin{tabular}{|c|c|c|c|c|}
\hline \multirow{2}{*}{ Sujet } & \multirow{2}{*}{$\begin{array}{c}\text { Date } \\
\text { de } \\
\text { prélèvement }\end{array}$} & \multirow{2}{*}{$\begin{array}{c}\text { Créatinine } \\
(\mathrm{g} / \mathrm{l})\end{array}$} & \multicolumn{2}{|c|}{ Uranium naturel } \\
\hline & & & $\mu \mathrm{g} / \mathrm{l}$ & $\begin{array}{c}\text { Créatinine } \\
(\mu \mathrm{g} / \mathrm{g})\end{array}$ \\
\hline $\mathrm{AY}, \ldots \ldots \ldots \ldots$ & $\begin{array}{l}11.02 .78 \\
13.02 .78\end{array}$ & $\begin{array}{l}0: 41 \\
2: 48\end{array}$ & $\begin{array}{r}8 \\
10\end{array}$ & $\begin{array}{c}19.5 \\
4\end{array}$ \\
\hline $\operatorname{RE} \ldots \ldots \ldots \ldots \ldots$ & $\begin{array}{l}03.02 .78 \\
05.02 .78\end{array}$ & $\begin{array}{l}3,97 \\
4,06\end{array}$ & $\begin{array}{l}23 \\
19\end{array}$ & $\begin{array}{l}5.8 \\
4.7\end{array}$ \\
\hline RA... & $\begin{array}{l}10.01 .78 \\
12.01 .78\end{array}$ & $\begin{array}{l}0,58 \\
2,50\end{array}$ & $\begin{array}{l}25 \\
27\end{array}$ & $\begin{array}{l}43 \\
11\end{array}$ \\
\hline
\end{tabular}

De même, chez le sujet RE : Taux de créatinine urinaire élevés $(3,97$ et 4,06 ). Lorsqu'on examine l'uranium rapporté au gramme de créatinine, l'excrétion en uranium est faible. Ce sujet devait avoir une diurèse de 24 heures faible.

Par ailleurs, nous avons pu constater que les taux d'élimination urinaire de l'uranium varient suivant l'heure de prélèvement. C'est ainsi que nous avons recueilli les urines de 24 heures chez plusieurs travailleurs : à chaque émission d'urine correspondait un flacon différent, analysé séparément. Nous avons constaté que, pour un même individu et suivant l'heure d'émission de la journée, on observait des variations (tableau II).

En ne considérant que l'excrétion urinaire, on observe une différence d'un facteur 25 environ pour les deux prélèvements de $15 \mathrm{~h} 15$ et $7 \mathrm{~h} 30$ alors qu'en réalité, rapporté à la créatinine, ce facteur n'est que de 2 . Ce cas extrême, choisi intentionnellement chez un agent qui n'éliminait que $600 \mathrm{ml} / \mathrm{j}$, met cependant en évidence que, pour un même sujet, l'excrétion urinaire de l'uranium n'est pas uniforme dans le nycthémère et qu'elle peut être sujette à des variations importantes.

\subsection{INTERPRÉTATION DES EXCRÉTIONS URINAIRES}

Outre les difficultés de prélèvement et les multiples causes d'erreurs susceptibles d'être rencontrées, l'interprétation des résultats est bien souvent 
délicate. Assurément, ce n'est pas à une seule valeur que nous pouvons faire confiance; c'est la raison pour laquelle il nous a paru préférable de faire une appréciation globale à partir des données ponctuelles que nous recueillons. Nous établissons des graphiques qui, étalés dans le temps, corrigent mieux les erreurs ou les variations individuelles de l'excrétion et permettent de mieux suivre l'évolution de l'imprégnation en uranium de tout travailleur exposé.

\section{TABLEAU II}

VARIATION, AU COURS DU NYCTHÉMERE, DE L'EXCRÉTION URINAIRE DE L'URANIUM

\begin{tabular}{|c|c|c|c|c|c|c|}
\hline \multirow{2}{*}{ Sujet } & \multirow{2}{*}{$\begin{array}{c}\text { Date } \\
\text { et } \\
\text { heure }\end{array}$} & \multirow{2}{*}{$\begin{array}{l}\text { Créatinine } \\
(\mathrm{g} / \mathrm{l})\end{array}$} & \multicolumn{2}{|c|}{ Uranium naturel } & \multirow{2}{*}{$\begin{array}{l}\text { Volume } \\
\text { d'urine } \\
\text { (ml/24 h) }\end{array}$} & \multirow{2}{*}{$\begin{array}{c}\text { Quantité } \\
\text { d'U } \\
\text { excrété } \\
(\mu \mathrm{g} / 24 \mathrm{~h})\end{array}$} \\
\hline & & & $(\mu \mathrm{g} / \mathrm{l})$ & $\begin{array}{l}\text { Créatinine } \\
(\mu \mathrm{g} / \mathrm{g})\end{array}$ & & \\
\hline \multirow[t]{12}{*}{ LU.... } & $08.01 .78:$ & & & & \multirow{12}{*}{660} & \multirow{12}{*}{25,4} \\
\hline & $11 \mathrm{~h} 10 \mathrm{~min}$ & 1,05 & 54 & 51,4 & & \\
\hline & $12 \mathrm{~h} 15 \mathrm{~min}$ & 1,07 & 50 & 46,7 & & \\
\hline & $15 \mathrm{~h} 15 \mathrm{~min}$ & 0,22 & 7 & 31,8 & & \\
\hline & $17 \mathrm{~h} 00 \mathrm{~min}$ & 0,24 & 7 & 29,2 & & \\
\hline & 19 h $30 \mathrm{~min}$ & 0,66 & 19 & 28,2 & & \\
\hline & $21 \mathrm{~h} 15 \mathrm{~min}$ & 0,65 & 17 & 26,1 & & \\
\hline & $23 \mathrm{~h} 15 \mathrm{~min}$ & 1,94 & 44 & 22,7 & & \\
\hline & $09.01 .78:$ & & & & & \\
\hline & $01 \mathrm{~h} 20 \mathrm{~min}$ & 1,95 & 38 & 19,5 & & \\
\hline & $06 \mathrm{~h} 20 \mathrm{~min}$ & 2,90 & 70 & 24,1 & & \\
\hline & $07 \mathrm{~h} 30 \mathrm{~min}$ & 2,42 & 160 & 66,1 & & \\
\hline
\end{tabular}

Le premier type de graphique, propre à chaque agent, concrétise son taux d'imprégnation; il est mis à jour très régulièrement. Deux courbes sont tracées : celle de l'excrétion urinaire mesurée en fin de poste de travail (uranium total absorbé), celle de l'excrétion urinaire mesurée en début de poste de travail (uranium total absorbé), celle de l'excrétion urinaire mesurée en début de poste de travail (uranium résiduel). C'est ce deuxième tracé dont nous tenons principalement compte; dans l'hypothèse la plus défavorable, il nous amènerait à retirer le travailleur de son poste et à entreprendre des examens plus approfondis ( fig. 1).

Le deuxième type de graphique est propre au poste de travail. Il est le reflet de l'imprégnation urinaire moyenne du personnel à un poste donnié Étudié en parallèle avec les courbes moyennes de concentration de l'air

vol. $15-\mathrm{N}^{\circ} 1$ 
ambiant, il permet de cerner de plus près les nuisances aux divers postes considérés ( fig. 2).

Le troisième type de graphique est établi pour chaque atelier. Il associe la courbe moyenne d'élimination de l'uranium urinaire pour un poste considéré à la courbe moyenne de concentration atmosphérique des poussières au même poste ( fig. 3).

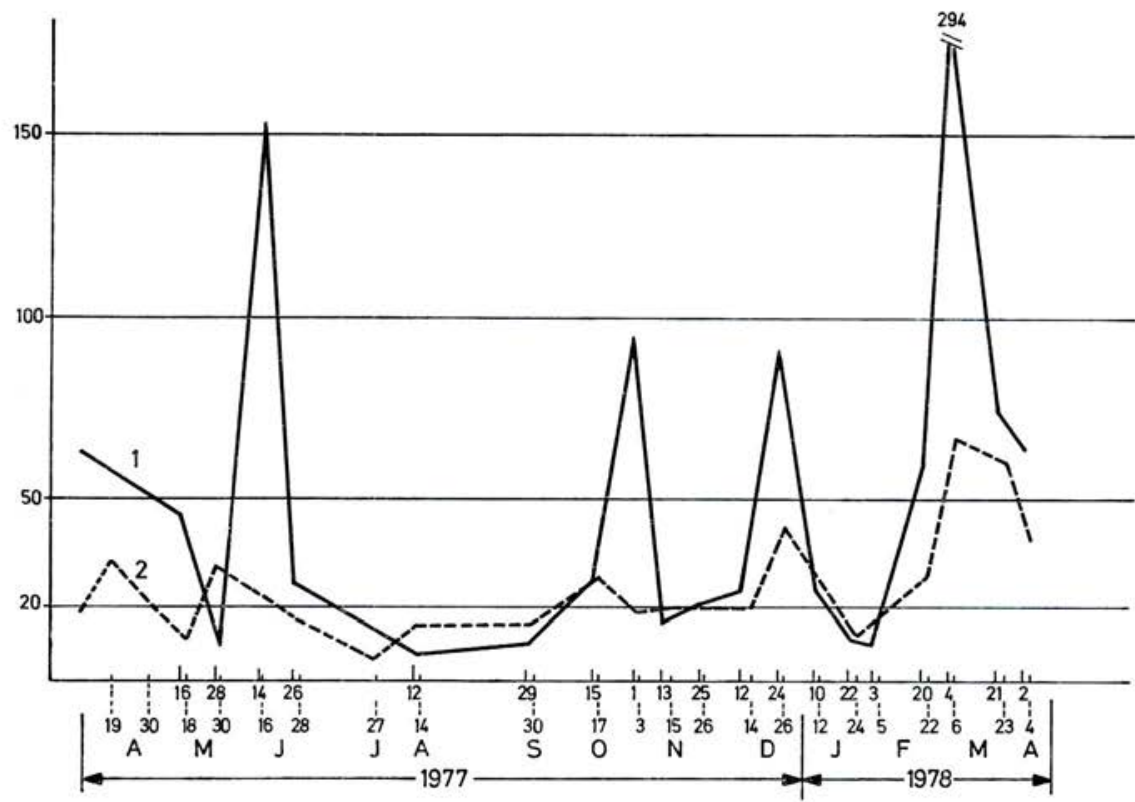

FIG. 1. - Excrétion urinaire $(\mu \mathrm{g} / \mathrm{l})$ des travailleurs fabriquant $\mathrm{UF}_{4}$ : pics des transférables du 19 avril 1977 au 4 avril 1978. Courbe 1 : prélèvement d'urine en fin de poste, cette courbe rend compte des pics dus à la présence de solubles $\mathrm{UO}_{2} \mathrm{~F}_{2}$. Courbe 2 : prélèvement d'urine avant la reprise de poste.

L'exploitation de ces différents graphiques confirme l'impression que nous avions au début : lorsqu'une courbe d'excrétion urinaire présente un pic élevé, le retour dans les zones traduisant une faible imprégnation est très rapide.

De même, l'élimination totale des composés moyennement transférables ( $\mathrm{UO}_{3}, \mathrm{UF}_{4}$, uranates) paraît correspondre aux valeurs proposées par la C.I.P.R. (de l'ordre de quelques semaines). Ainsi, la création par la C.I.P.R. de la classe de composés moyennement transférables nous paraît très justifiée. Par contre, en ce qui concerne les composés peu transférables $\left(\mathrm{U}_{3} \mathrm{O}_{8}\right)$ que nous traitons à l'usine, il nous semble que leur période d'épuration biologique est nettement inférieure à $500 \mathrm{j}$. 


\section{CONTRÔLE DE L'AMBIANCE GÉNÉRALE DES ATELIERS}

L'étude des courbes d'excrétion urinaire est complétée par la surveillance permanente des concentrations atmosphériques des ateliers; elle nous renseigne journellement sur l'évolution de la pollution des lieux de travail et nous guide pour déterminer la fréquence des prélèvements urinaires.

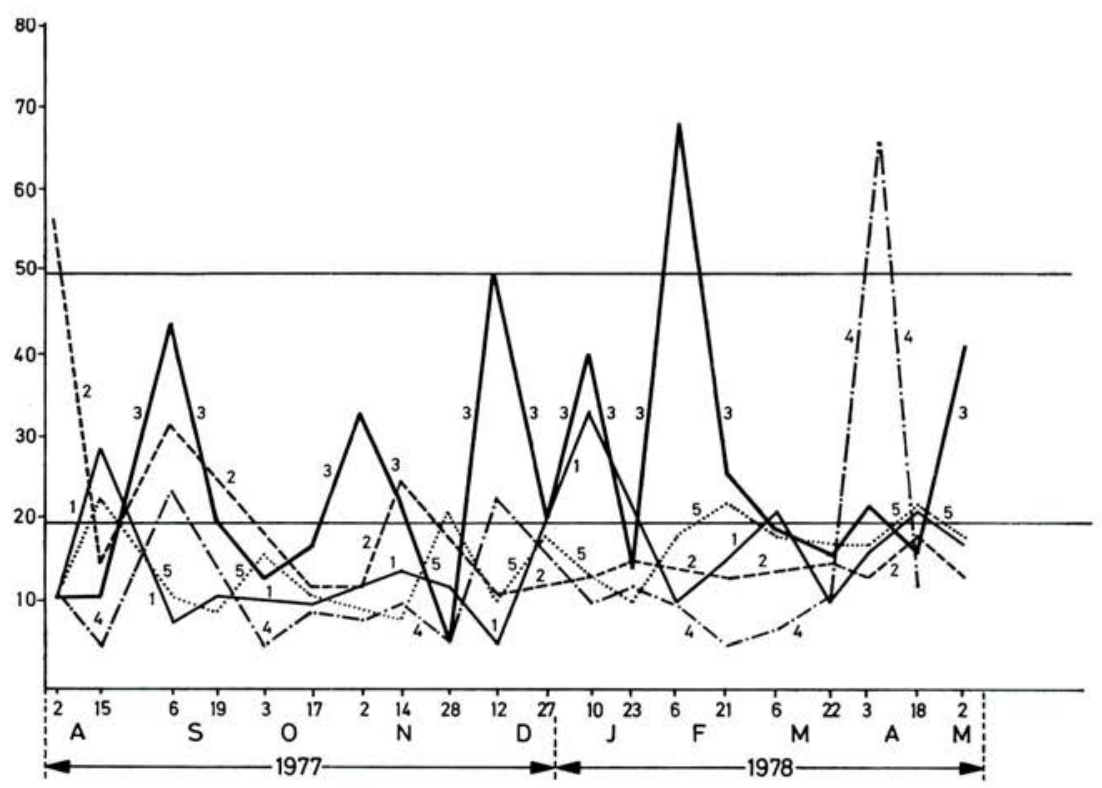

FIG. 2. - Type de graphique pour un poste de travail : «Fluoration bas », 1 à 2 personnes par poste; 5 équipes (1) à (5). Excrétion urinaire $(\mu \mathrm{g} / \mathrm{l})$ d'UF $_{4}$ et $\mathrm{UO}_{2} \mathrm{~F}_{2}$ du 2 août 1977 au 2 mai 1978.

La surveillance des ambiances de travail comporte trois types de contrôles : celui de l'ambiance générale d'un atelier; celui des postes de travail; les contrôles individuels.

\subsection{Les ambiances géNÉRALES DE L'ATElier}

La mesure est fournie quotidiennement par le S.P.R. de l'usine; les comptages de poussières radioactives sont exprimés en picocuries $\left(60 \mathrm{pCi} / \mathrm{m}^{3}\right.$ étant l'activité maximale admissible retenue dans notre usine qui traite de l'uranium naturel).

\subsection{Contrôles des postes de travail}

Des dosages plus précis, exprimés en milligrammes d'uranium par mètre cube d'air, nous sont fournis mensuellement par le S.H.I. de Pierrelatte (concentration maximale admissible $=0,150 \mathrm{mg} / \mathrm{m}^{3}$ pour l'uranium naturel 
sous forme de composés non transférables). Ces dosages pondéraux complètent les mesures de radioactivité effectuées au poste du travailleur. Les prélèvements d'air ont une durée de $90 \mathrm{~min}$, avec un débit de 18001 d'air par heure.

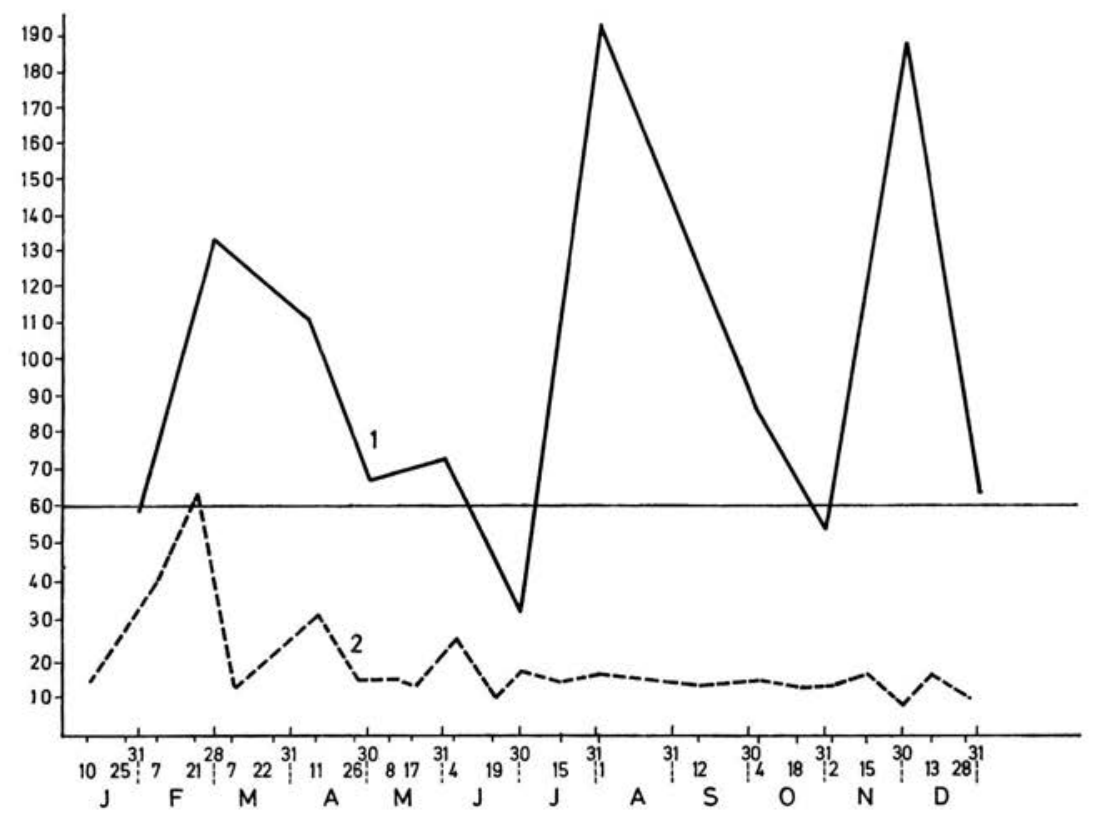

FIG. 3. - Atelier de dissolution du poste basculement, année 1977. (1) Courbes moyennes des concentrations atmosphériques $\left(\mathrm{Ci} / \mathrm{m}^{3}\right)$. (2) Excrétion urinaire en fin de poste $(\mu \mathrm{g} / 1)$. La courbe d'excrétion urinaire ne suit pas celle de la concentration atmosphérique, car l'ouvrier porte le masque épisodiquement et se déplace pendant son travail.

\subsection{Contrôle INDIVIDUELS}

Ils sont pratiqués à l'aide de pompes portatives (Casella), en fonction de certains besoins particuliers. L'appareil, fixé au revers de la veste, suit le travailleur dans tous ses déplacements. Il a un débit de $240 \mathrm{l} / \mathrm{h}$, la durée moyenne du prélèvement est 2 heures. Ces contrôles toujours intéressants demandent cependant à être interprétés : Le débit des appareils de prélèvement d'air n'est pas exactement le même que celui de l'appareil pulmonaire humain. De plus, si l'appareil aspire, il n'expire pas. Enfin, on connaît très bien l'influence des divers courants d'air, des vents dominants, de l'état hygrométrique de l'air et de la granulométrie des poussières considérées. Nous établissons ensuite, pour chaque atelier, le troisième type de graphique qui associe la courbe moyenne d'élimination de l'uranium urinaire, pour un poste considéré, à la courbe moyenne de concentration atmosphérique des poussières, au même poste (fig. 3). L'étude de ces graphiques permet une meilleure approche de la contamination interne, en fonction des concentrations atmos- 
phériques et des conclusions à en tirer pour une amélioration des conditions de travail. Nous avons pu ainsi, par cette étude, préciser quelques notions importantes :

- les concentrations atmosphériques varient dans le temps;

- elles varient dans l'espace;

- les moyens de protection individuelle (masques en particulier) sont très efficaces.

\section{5. ÉTUDE PHYSICO-CHIMIQUE DES COMPOSÉS D'URANIUM INHALÉS}

Indépendamment des mesures de concentrations globale dans l'air, nous déterminons la solubilité de l'uranium dans l'eau; nous le prélevons dans ce but, soit sur les filtres des aspirateurs individuels, soit dans les conteneurs, soit sur les parois des ateliers.

Par cet examen relativement simple, nous faisons des observations particulièrement intéressantes à double titre.

5.1. On s'aperçoit parfois que tel composé réputé peu soluble dans la littérature pouvait être de solubilité différente dans un atelier de fabrication selon la bonne ou la mauvaise marche de celui-ci; c'est ainsi que sur deux échantillons différents de tétrafluorure d'uranium, la solubilité était de 9 fois environ la valeur théorique du premier et de 20 fois celle du deuxième (tableau III).

\section{TABLEAU III}

ÉTUDE DE LA SOLUBILITÉ EFFECTIVE DES COMPOSÉS D'URANIUM SE TROUVANT DANS NOTRE USINE

\begin{tabular}{|c|c|c|}
\hline $\begin{array}{l}\text { Composés } \\
\text { testés }\end{array}$ & $\begin{array}{c}\text { Indication fournie } \\
\text { par la } \\
\text { littérature } \\
(\mathrm{mg} / \mathrm{l})\end{array}$ & $\begin{array}{c}\text { Solubilisation } \\
\text { expérimentale } \\
(\mathrm{mg} / \mathrm{l})\end{array}$ \\
\hline Uranates d'ammonium..... & Insoluble & 1,6 \\
\hline Oxyde $\mathrm{U}_{3} \mathrm{O}_{8} \ldots \ldots \ldots \ldots$ & Insoluble & 1,8 \\
\hline Tétrafluorure............. & 31,4 & $\begin{array}{c}286 \\
\text { sur l'échantillon A } \\
600 \\
\text { sur l'échantillon B }\end{array}$ \\
\hline Oxyde $\mathrm{UO}_{3} \ldots \ldots \ldots \ldots$ & 160 & 3,5 \\
\hline
\end{tabular}


On peut expliquer ces valeurs surprenantes par la présence d'oxyfluorure d'uranium $\left(\mathrm{UO}_{2} \mathrm{~F}_{2}\right)$ en proportion importante dans le tétrafluorure. Cette hypothèse est tout à fait compatible avec le procédé de fabrication de l'UF $_{4}$. L'importante proportion d'oxyfluorure (composé transférable au plan biologique) dans la poudre de tétrafluorure (composé moyennement transférable) permet d'expliquer les variations observées dans l'excrétion urinaire et l'apparente contradiction avec les données fournies par la littérature.

5.2. Ces variations dans la composition réelle de nos fabrications expliquent les pics à éliminations très rapide des courbes de fin de poste. Elles rendent aussi compte de la difficulté éprouvée dans le travail en usine à bien séparer les différents types de composés uranifères.

\section{CONCLUSIONS}

6.1. Après avoir résolu un certain nombre de difficultés, nous sommes donc parvenus à obtenir une appréciation correcte de la mesure de l'uranium urinaire. Ce dosage, méthode indirecte de mesure de la contamination interne, garde actuellement à nos yeux toute sa valeur dans la surveillance de routine des travailleurs exposés aux composés uranifères. Il est, cependant, nécessaire de prendre un certain nombre de précautions pour obtenir une réponse correcte : prélèvements à domicile évitant tout apport exogène; homogénéisation de l'échantillon avant l'analyse; mesure de la créatinine.

Les résultats doivent ensuite être interprétés sous certaines conditions : (a) appréciation de la contamination par l'examen de graphiques individuels d'excrétion urinaire : un résultat isolé a peu de signification, et doit être replacé dans son contexte; $(b)$ association des graphiques individuels à la surveillance régulière des ambiances de travail; $(c)$ étude de la solubilité physico-chimique des composés fabriqués à l'usine (pics des transférables).

Cette méthode relativement lourde et difficile à mettre en œuvre nous paraît, cependant, être la seule valable actuellement pour apprécier une contamination interne, toujours difficile à déterminer.

Nous espérons pouvoir la compléter ou la remplacer par des méthodes plus simples ou plus directes (dosages dans les fèces ou anthropogammamétrie par exemple).

6.2. Elle nous a permis cependant de dégager plusieurs notions qui nous ont paru très importantes :

- nous avons dû, en premier lieu, nous rendre compte qu'il était difficile, dans le travail en usine, de bien différencier les composés transférables de ceux moyennement transférables ou peu transférables. Dans une usine ne traitant théoriquement que des composés moyennement ou peu transférables, on peut rencontrer, en cours de fabrication, des composés transférables, ce qui implique une surveillance du personnel comme s'il était 
normalement exposé à ces composés, en particulier, dans la surveillance de la fonction rénale;

- Ainsi, nous avons pu avoir une idée plus précise, bien qu'encore incertaine de la durée d'élimination des différents composés au stade de l'usine :

- pour les composés transférables, elles dépassent rarement $48 \mathrm{~h}$, comme nous avons pu le vérifier lors d'inhalations importantes d'UF ${ }_{6}$ dans une autre usine;

- de même, nous nous sommes rendu compte que la nouvelle classification introduisant la notion de composés moyennement transférables (classe W), à période biologique d'élimination de 50 jours environ, est beaucoup plus proche de la réalité que l'ancienne qui n'en tenait pas compte $\left(\mathrm{UO}_{3}, \mathrm{UF}_{4}\right)$;

- enfin, nous nous demandons si la période biologique d'élimination des composés peu transférables est aussi longue que ce qu'on pourrait penser $(500 \mathrm{j})$. Cette notion demande à être approfondie et discutée.

6.3. Nous pensons compléter cette étude lorsque nous serons en possession de résultats obtenus après dosage de l'uranium dans les selles ou par anthropogammamétrie, procédés pour lequel notre expérience est encore trop limitée. En outre, nous envisageons d'établir des courbes théoriques d'élimination de l'uranium à partir de nos résultats urinaires et dès que nous le pourrons, des résultats fécaux et anthropogammamétriques. Nous espérons pouvoir appréhender ainsi, d'une façon plus précise, la période biologique effective des poussières des composés inhalés, ce qui, en réalité, conditionne et doit conditionner les normes de surveillance des travailleurs.

\section{BIBLIOGRAPHIE}

[1] Chalabreysse J. Étude et résultats d'examens effectués à la suite d'une inhalation de composés dits solubles d'uranium naturel. Radioprotection, 1970, 5, (1), 1-17; 5, (4), 305-310.

[2] ChalabreysSe J. Toxicologie de l'uranium naturel, essai d'évaluation de la contamination chez l'homme. Rapport CEA-R-3361, 1968.

[3] Chalabreysse J. Surveillance radiotoxicologique du personnel de l'usine de séparation des isotopes de l'uranium par diffusion gazeuse de Pierrelatte, Thèse, Lyon, 1976; Rapport COGEMA-R-2, 1978.

[4] Chalabreysse J. Surveillance radiotoxicologique du personnel de l'usine de séparation des isotopes de l'uranium de Pierrelatte, Méthodes et résultats, I.R.P.A. 4. Congrès international, Paris, 24-30 avril 1977, $\mathrm{n}^{\circ}$ 252, 1, 45-48. 